Ahmed Jamea (El Jadida)

Abderrahmane El Hachimi (Rabat)

JAOUAD IGBIDA (El Jadida)

\title{
UNIQUENESS OF RENORMALIZED SOLUTION TO NONLINEAR NEUMANN PROBLEMS WITH VARIABLE EXPONENT
}

Abstract. We study the uniqueness of renormalized solutions to nonlinear Neumann problems with variable exponents

$$
\begin{cases}|u|^{p(x)-2} u-\Delta_{p(x)}(u)=f & \text { in } \Omega, \\ |\nabla u|^{p(x)-2} \frac{\partial u}{\partial \eta}+\gamma(u)=g & \text { on } \partial \Omega,\end{cases}
$$

where $\Omega$ is a connected open bounded set in $\mathbb{R}^{N}, p(\cdot)$ is a continuous function defined on $\bar{\Omega}$ with $p(x)>1$ for all $x \in \bar{\Omega}, \gamma$ is a nondecreasing continuous function on $\mathbb{R}$ such that $\gamma(0)=0$ and $f, g \in L^{1}$.

1. Introduction. In the present paper, we prove a uniqueness result for renormalized solutions to the nonlinear Neumann problem with variable exponent

$$
\begin{cases}|u|^{p(x)-2} u-\Delta_{p(x)}(u)=f & \text { in } \Omega, \\ |\nabla u|^{p(x)-2} \frac{\partial u}{\partial \eta}+\gamma(u)=g & \text { on } \partial \Omega,\end{cases}
$$

where $p(\cdot)$ is a continuous function defined on $\bar{\Omega}$ with $p(x)>1$ for all $x \in \bar{\Omega}$, $\Omega$ is a connected open bounded set in $\mathbb{R}^{N}, N \geq 3$, with a connected Lipschitz boundary $\partial \Omega, \eta$ is the unit outward normal on $\partial \Omega$, and $\gamma$ is a nondecreasing

2010 Mathematics Subject Classification: Primary 35J60; Secondary 35A02, 35J66, 35J70. Key words and phrases: nonlinear elliptic problem, Neumann-type boundary condition, renormalized solution, variable exponent, uniqueness.

Received 29 December 2015; revised 8 June 2016.

Published online 21 October 2016. 
continuous function on $\mathbb{R}$ such that $\gamma(0)=0$. We will have in mind especially the case when the right-hand sides lie in $L^{1}$.

The operator

$$
\Delta_{p(x)} u=\operatorname{div}\left(|\nabla u|^{p(x)-2} \nabla u\right)
$$

is called the $p(x)$-Laplacian, which becomes the $p$-Laplacian when $p(x) \equiv p$ (a constant).

In recent years, there is a lot of interest in various mathematical problems with variable exponent (see for example [7, 8, 18, 21, 31, 30] and references therein). Such problems are also interesting in applications. They appear in models of electrorheological fluids, of stationary thermo-rheological viscous flows of non-Newtonian fluids and in the mathematical description of filtration of an ideal barotropic gas through a porous medium. We refer the reader for example to [13, 21, 28] and references therein for more details.

We recall that the notion of renormalized solution was introduced by DiPerna and Lions 16 in their study of the Boltzmann equation; this notion was then adapted by many authors to study some nonlinear elliptic problems in the case where $p(\cdot)=p$ (a constant) with Dirichlet or Neumann boundary conditions and for the corresponding parabolic equations with $L^{1}$ data, (see for example [1, 3, 10, 11, 17, 25, 26, 27, 29]). We also recall that this type of solutions is equivalent to another concept of solutions, called entropy solutions, introduced independently by Bénilan et al. [9].

This paper is organized as follows. In Section 2, we fix the notation and give some preliminaries. In Section 3, following [7, 26, 30], we introduce the concept of renormalized solution for $(1.1)$ and state the uniqueness result for this type of solution. We recall that in the case where the exponent variable $p(\cdot)$ is a bounded continuous function on $\bar{\Omega}$, the existence of a renormalized solution to this type of problem was studied by E. Azroul et al. [7].

The case where $p(\cdot)$ is unbounded has recently been considered by several authors (see for example [14, 20, 22, 24, and references therein). The Neumann nonhomogeneous boundary problem

$$
\begin{cases}\Delta_{p(x)}(u)=0 & \text { in } \Omega, \\ |\nabla u|^{p(x)-2} \frac{\partial u}{\partial \eta}=h & \text { on } \partial \Omega,\end{cases}
$$

where $p(x) \equiv \infty$ in a subdomain of $\Omega, h \in C(\bar{\Omega})$ and $\int_{\partial \Omega} h=0$, was analyzed by Y. Karagiorgos and N. Yannakakis [22]. Problem (1.2) with Dirichlet boundary condition and $p(x) \equiv \infty$ in a subdomain of $\Omega$ was analyzed by J. J. Manfredi, J. D. Rossi and J. M. Urbano [24].

In general, the properties of Lebesgue and Sobolev spaces with unbounded variable exponent are more complex than in the case of bounded ex- 
ponent (see for example [15] for definitions and basic properties of Lebesgue and Sobolev spaces with unbounded variable exponent).

2. Preliminaries and notation. In this section, we give some notation, definitions and results that we use in this work.

Let $\Omega$ be a measurable connected open bounded set in $\mathbb{R}^{N}, N \geq 3$; let meas $(\Omega)$ denote its measure. We write $C^{+}(\bar{\Omega})=\left\{\right.$ continuous functions $p(\cdot): \bar{\Omega} \rightarrow \mathbb{R}^{+}$such that $\left.1<p_{-}<p_{+}<\infty\right\}$, where

$$
p_{-}=\min _{x \in \bar{\Omega}} p(x) \quad \text { and } \quad p_{+}=\max _{x \in \bar{\Omega}} p(x) .
$$

For $p(\cdot) \in C^{+}(\bar{\Omega})$, we define the Lebesgue space with variable exponent $L^{p(\cdot)}(\Omega)$ by

$$
L^{p(\cdot)}(\Omega)=\left\{u: \Omega \rightarrow \mathbb{R} \text { measurable }: \int_{\Omega}|u(x)|^{p(x)} d x<\infty\right\},
$$

endowed with the Luxemburg norm

$$
\|u\|_{p(\cdot)}=\|u\|_{L^{p(\cdot)}(\Omega)}=\inf \left\{\lambda>0, \int_{\Omega}\left|\frac{u(x)}{\lambda}\right|^{p(x)} d x \leq 1\right\} .
$$

The space $\left(L^{p(\cdot)}(\Omega),\|\cdot\|_{p(\cdot)}\right)$ is a reflexive, uniformly convex Banach space, and its dual space is isomorphic to $L^{p^{\prime}(\cdot)}(\Omega)$ where $1 / p(\cdot)+1 / p^{\prime}(\cdot)=1$ (see [19]).

Proposition 2.1 (Hölder type inequality [19]). Let $p(\cdot), p^{\prime}(\cdot) \in C^{+}(\bar{\Omega})$ with $1 / p(\cdot)+1 / p^{\prime}(\cdot)=1$. Then for any $u \in L^{p(\cdot)}(\Omega)$ and $v \in L^{p^{\prime}(\cdot)}(\Omega)$ we have

$$
\left|\int_{\Omega} u \cdot v d x\right| \leq\left(\frac{1}{p_{-}}+\frac{1}{p_{-}^{\prime}}\right)\|u\|_{p(\cdot)}\|u\|_{p^{\prime}(\cdot)} .
$$

We also consider the function $\rho_{p(\cdot)}: L^{p(\cdot)}(\Omega) \rightarrow \mathbb{R}$ defined by

$$
\rho_{p(\cdot)}(u)=\rho_{L^{p(\cdot)}(\Omega)}(u)=\int_{\Omega}|u(x)|^{p(x)} d x .
$$

The connection between $\rho_{p(\cdot)}$ and $\|\cdot\|_{p(\cdot)}$ is established by the next result.

Proposition 2.2 (Fan and Zhao [19]).

(a) Let $u \in L^{p(\cdot)}(\Omega)$. We have

(i) $\|u\|_{p(\cdot)}<1$ (respectively $\left.>,=1\right) \Leftrightarrow \rho_{p(\cdot)}(u)<1$ (respectively $>,=1)$.

(ii) $\|u\|_{p(\cdot)}=\alpha \Leftrightarrow \rho_{p(\cdot)}(u)=\alpha($ when $\alpha \neq 0)$. 
(iii) If $\|u\|_{p(\cdot)}<1$ then $\|u\|_{p(\cdot)}^{p_{+}} \leq \rho_{p(\cdot)}(u) \leq\|u\|_{p(\cdot)}^{p_{-}}$.

(iv) If $\|u\|_{p(\cdot)}>1$ then $\|u\|_{p(\cdot)}^{p_{-}} \leq \rho_{p(\cdot)}(u) \leq\|u\|_{p(\cdot)}^{p_{+}}$.

(b) For a sequence $\left(u_{n}\right)_{n \in \mathbb{N}} \subset L^{p(\cdot)}(\Omega)$ and $u \in L^{p(\cdot)}(\Omega)$, the following statements are equivalent:

(i) $\lim _{n \rightarrow \infty} u_{n}=u$ in $L^{p(\cdot)}(\Omega)$.

(ii) $\lim _{n \rightarrow \infty} \rho_{p(\cdot)}\left(u_{n}-u\right)=0$.

(iii) $u_{n} \rightarrow u$ in measure in $\Omega$.

The variable exponent Sobolev space $W^{1, p(\cdot)}(\Omega)$ consists of all $u \in$ $L^{p(\cdot)}(\Omega)$ such that the absolute value of the gradient is in $L^{p(\cdot)}(\Omega)$. Let

$$
\|u\|_{1, p(\cdot)}=\|u\|_{p(\cdot)}+\|\nabla u\|_{p(\cdot)} .
$$

Then $\left(W^{1, p(\cdot)}(\Omega),\|\cdot\|_{1, p(\cdot)}\right)$ is a separable and reflexive Banach space. For a given constant $k>0$, we define $T_{k}: \mathbb{R} \rightarrow \mathbb{R}$ as

$$
T_{k}(s):= \begin{cases}s & \text { if }|s| \leq k, \\ k \operatorname{sign}(s) & \text { if }|s|>k,\end{cases}
$$

where

$$
\operatorname{sign}(s):= \begin{cases}1 & \text { if } s>0, \\ 0 & \text { if } s=0, \\ -1 & \text { if } s<0 .\end{cases}
$$

And for a function $u=u(x)$ defined on $\Omega$, we define the truncated function $T_{k} u$ by setting $\left(T_{k} u\right)(x)=T_{k}(u(x))$ for every $x \in \Omega$.

We also define the space

$$
\mathcal{T}^{1, p(\cdot)}(\Omega)=\left\{u: \Omega \rightarrow \mathbb{R} \text { measurable: } T_{k}(u) \in W^{1, p(\cdot)}(\Omega) \text { for all } k>0\right\} .
$$

By [9], we have the following result:

Proposition 2.3. For every $u \in \mathcal{T}^{1, p(\cdot)}(\Omega)$, there exists a unique measurable function $v: \Omega \rightarrow \mathbb{R}^{N}$ such that

$$
\nabla T_{k}(u)=\chi_{\{|u| \leq k\}} \nabla v \quad \text { for all } k>0,
$$

where $\chi_{B}$ is the characteristic function of the measurable set $B \subset \mathbb{R}^{N}$. The function $v$ is denoted by $\nabla u$.

Moreover, if $u \in W^{1, p(\cdot)}(\Omega)$, then $v \in\left(L^{p(\cdot)(\Omega)}\right)^{N}$ and $v=\nabla u$ in the usual sense.

For $u \in W^{1, p(\cdot)}(\Omega)$, we denote by $\tau u$ or $u$ the trace of $u$ on $\partial \Omega$ in the usual sense.

On the other hand, as in [4], $\mathcal{T}_{\mathrm{tr}}^{1, p(\cdot)}(\Omega)$ denotes the set of functions $u \in \mathcal{T}^{1, p(\cdot)}(\Omega)$ which satisfy the following condition: There exists a sequence $\left(u_{n}\right)_{n \in \mathbb{N}}$ in $W^{1, p(\cdot)}(\Omega)$ and a measurable function $v$ on $\partial \Omega$ such that 
(a) $u_{n} \rightarrow u$ a.e. in $\Omega$,

(b) $\nabla T_{k}\left(u_{n}\right) \rightarrow \nabla T_{k}(u)$ in $\left(L^{1}(\Omega)\right)^{N}$ for every $k>0$,

(c) $u_{n} \rightarrow v$ a.e. on $\partial \Omega$.

The function $v$ is the trace of $u$ in the generalized sense. For $u \in \mathcal{T}_{\mathrm{tr}}^{1, p(\cdot)}(\Omega)$, the trace of $u$ on $\partial \Omega$ is denoted by $\operatorname{tr}(u)$ or $u$. The operator $\operatorname{tr}(\cdot)$ has the following properties [4]:

(i) If $u \in \mathcal{T}_{\operatorname{tr}}^{1, p(\cdot)}(\Omega)$, then $\tau T_{k}(u)=T_{k}(\operatorname{tr}(u))$ for all $k>0$,

(ii) if $\varphi \in W^{1, p(\cdot)}(\Omega) \cap L^{\infty}(\Omega)$, then for all $u \in \mathcal{T}_{\operatorname{tr}}^{1, p(\cdot)}(\Omega)$, we have $u-\varphi \in \mathcal{T}_{\mathrm{tr}}^{1, p(\cdot)}(\Omega)$ and $\operatorname{tr}(u-\varphi)=\operatorname{tr}(u)-\tau \varphi$.

In the case where $u \in W^{1, p(\cdot)}(\Omega), \operatorname{tr}(u)$ coincides with $\tau u$.

Obviously, we have

$$
W^{1, p(\cdot)}(\Omega) \subset \mathcal{T}_{\operatorname{tr}}^{1, p(\cdot)}(\Omega) \subset \mathcal{T}^{1, p(\cdot)}(\Omega) .
$$

3. Assumptions and statement of the uniqueness result. In this section, we give the concept of renormalized solution for problem (1.1) and state the uniqueness result for this type of solution. We assume the following hypotheses.

$\left(\mathrm{H}_{1}\right) f \in L^{1}(\Omega)$ and $g \in L^{1}(\partial \Omega)$ are positive functions.

$\left(\mathrm{H}_{2}\right) \gamma$ is a nondecreasing continuous function on $\mathbb{R}$ such that $\gamma(0)=0$.

Now, we extend the notion of entropy solution to problem (1.1):

Definition 3.1. A measurable function $u: \Omega \rightarrow \mathbb{R}$ is a renormalized solution of the elliptic problem (1.1) if

$$
\begin{gathered}
u \in \mathcal{T}_{\operatorname{tr}}^{1, p(\cdot)}(\Omega), \\
\lim _{m \rightarrow \infty} \frac{1}{m} \int_{\{m \leq|u| \leq 2 m\}}|\nabla u|^{p(x)} d x=0,
\end{gathered}
$$

and

$$
\begin{aligned}
\int_{\Omega}|u|^{p(x)-2} u S(u) \varphi d x+\int_{\Omega}|\nabla u|^{p(x)-2} \nabla & u \nabla(S(u) \varphi) d x \\
& +\int_{\partial \Omega} \gamma(u) S(u) \varphi d \sigma \\
= & \int_{\Omega} f S(u) \varphi d x+\int_{\partial \Omega} g S(u) \varphi d \sigma
\end{aligned}
$$

for every $\varphi \in W^{1, p(\cdot)}(\Omega) \cap L^{\infty}(\Omega)$ and every $S \in W^{1, \infty}(\Omega)$ such that $\operatorname{supp}(S)$ is compact in $\mathbb{R}$. 
THEOREM 3.2. Let hypotheses $\left(\mathrm{H}_{1}\right)-\left(\mathrm{H}_{2}\right)$ hold. If $u$ is a renormalized solution of the nonlinear elliptic problem (1.1), then

$$
\begin{gathered}
\|u\|_{p(\cdot)-1}<\infty, \\
\frac{1}{m} \int_{\{|u| \leq m\}}|\nabla u|^{p(x)} d x \leq c(f ; g) \quad \text { for all } m>0, \\
\lim _{m \rightarrow \infty} \int_{\{m \leq|u| \leq m+k\}}|\nabla u|^{p(x)} d x=0 \quad \text { for all } k>0,
\end{gathered}
$$

where $c(f ; g)$ is a positive constant depending only on the data $f$ and $g$.

Proof. To prove (3.4) and (3.5), let $m>0$ and define

$$
S_{m}(t)= \begin{cases}1, & |t| \leq m, \\ 2-|t| / m, & m \leq|t| \leq 2 m, \\ 0, & |t| \geq 2 m .\end{cases}
$$

Let $k, m>0$. Taking $\varphi=T_{k}(u)$ and $S=S_{m}$ in (3.3), we obtain

$$
\begin{aligned}
\int_{\Omega}|u|^{p(x)-2} u S_{m}(u) T_{k}(u) d x & +\int_{\Omega}|\nabla u|^{p(x)-2} \nabla u \nabla\left(S_{m}(u) T_{k}(u)\right) d x \\
& +\int_{\partial \Omega} \gamma(u) S_{m}(u) T_{k}(u) d \sigma \\
= & \int_{\Omega} f S_{m}(u) T_{k}(u) d x+\int_{\partial \Omega} g S_{m}(u) T_{k}(u) d \sigma .
\end{aligned}
$$

This implies that

$$
\begin{aligned}
\int_{\Omega}|u|^{p(x)-2} u S_{m}(u) & T_{k}(u) d x+\int_{\{|u| \leq k\}}|\nabla u|^{p(x)} S_{m}(u) d x \\
& +\int_{\partial \Omega} \gamma(u) S_{m}(u) T_{k}(u) d \sigma \\
\leq & \frac{k}{m} \int_{\{m \leq|u| \leq 2 m\}}|\nabla u|^{p(x)} d x+k\left(\|f\|_{1}+\|g\|_{L^{1}(\partial \Omega)}\right),
\end{aligned}
$$

i.e.

$$
\begin{aligned}
\int_{\Omega}|u|^{p(x)-2} u S_{m}(u) & \frac{T_{k}(u)}{k} d x+\frac{1}{k} \int_{\{|u| \leq k\}}|\nabla u|^{p(x)} S_{m}(u) d x \\
\leq & \frac{1}{m} \int_{\{m \leq|u| \leq 2 m\}}|\nabla u|^{p(x)} d x+\|f\|_{1}+\|g\|_{L^{1}(\partial \Omega)} .
\end{aligned}
$$


Hence

$$
\int_{\Omega}|u|^{p(x)-2} u S_{m}(u) \frac{T_{k}(u)}{k} d x \leq \frac{1}{m} \int_{\{m \leq|u| \leq 2 m\}}|\nabla u|^{p(x)} d x+\|f\|_{1}+\|g\|_{L^{1}(\partial \Omega)} .
$$

After taking the limit as $m \rightarrow \infty, k \rightarrow 0$, by Fatou's lemma, Definition 3.1 and the fact that

$$
\lim _{m \rightarrow \infty} S_{m}(t)=1, \quad \lim _{k \rightarrow 0} \frac{T_{k}(t)}{k}=\operatorname{sign}(t), \quad \text { for all } t \in \mathbb{R},
$$

we deduce that

$$
\int_{\Omega}|u|^{p(x)-1} d x \leq\|f\|_{1}+\|g\|_{L^{1}(\partial \Omega)} .
$$

Then by Proposition 2.2 we get

$$
\|u\|_{p(\cdot)-1}<\infty .
$$

On the other hand, inequality $(3.9)$ implies that

$$
\begin{aligned}
& \frac{1}{k} \int_{\{|u| \leq k\}}|\nabla u|^{p(x)} S_{m}(u) d x \\
& \quad \leq \frac{1}{m} \int_{\{m \leq|u| \leq 2 m\}}|\nabla u|^{p(x)} d x+\|f\|_{1}+\|g\|_{L^{1}(\partial \Omega)} .
\end{aligned}
$$

Passing the limit $m \rightarrow \infty$, we use (3.10), Fatou's lemma and $(3.2)$ to obtain

$$
\frac{1}{k} \int_{\{|u|<k\}}|\nabla u|^{p(x)} d x \leq c(f ; g) \quad \text { for all } k>0 .
$$

We now prove (3.6). Inserting $\varphi=T_{k}\left(u-T_{m}(u)\right), k>0$ and $S=S_{m}$ in (3.3) gives

$$
\begin{aligned}
& \int_{\Omega}|u|^{p(x)-2} u S_{m}(u) T_{k}\left(u-T_{m}(u)\right) d x \\
& \left.\quad+\int_{\Omega}|\nabla u|^{p(x)-2} \nabla u \nabla\left(S_{m}(u) T_{k}\left(u-T_{m}(u)\right)\right)\right) d x \\
& \quad+\int_{\partial \Omega} \gamma(u) S_{m}(u) T_{k}\left(u-T_{m}(u)\right) d \sigma \\
& =\int_{\Omega} f S_{m}(u) T_{k}\left(u-T_{m}(u)\right) d x+\int_{\partial \Omega} g S_{m}(u) T_{k}\left(u-T_{m}(u)\right) d \sigma .
\end{aligned}
$$

On the one hand, we have

$$
\begin{aligned}
I_{k ; m}^{1} & =\int_{\Omega}|u|^{p(x)-2} u S_{m}(u) T_{k}\left(u-T_{m}(u)\right) d x \\
& =\int_{\{|u|>m\}}|u|^{p(x)-2} u S_{m}(u) T_{k}(u-m \operatorname{sign}(u)) d x,
\end{aligned}
$$


since

$$
\operatorname{sign}(u) \chi_{\{|u|>m\}}=\operatorname{sign}\left(T_{k}(u-m \operatorname{sign}(u))\right) \chi_{\{|u|>m\}},
$$

thus

$$
I_{k ; m}^{1} \geq 0
$$

The same method yields

$$
\int_{\partial \Omega} \gamma(u) S_{m}(u) T_{k}\left(u-T_{m}(u)\right) d \sigma \geq 0 .
$$

On the other hand,

$$
\begin{aligned}
I_{k ; m}^{2}= & \int_{\Omega}|\nabla u|^{p(x)-2} \nabla u \nabla\left(S_{m}(u) T_{k}\left(u-T_{m}(u)\right)\right) d x \\
= & -\frac{1}{m} \int_{\{m \leq|u| \leq 2 m\}}|\nabla u|^{p(x)} \operatorname{sign}(u) T_{k}\left(u-T_{m}(u)\right) d x \\
& +\int_{\{m \leq|u| \leq m+k\}}|\nabla u|^{p(x)} S_{m}(u) d x .
\end{aligned}
$$

Therefore, 3.12 gives

$$
\begin{aligned}
\int_{\{m \leq|u| \leq m+k\}}|\nabla u|^{p(x)} & S_{m}(u) d x \\
\leq & \frac{k}{m} \int_{\{m \leq|u| \leq 2 m\}}|\nabla u|^{p(x)} d x+k \int_{\partial \Omega \cap\{\mid>m\}}|f| d x \\
& +k \int_{\partial|u|>m\}}|g| d \sigma .
\end{aligned}
$$

Now we pass to the limit in $(3.14)$ as $m \rightarrow \infty$ : we use $(3.2)$, hypothesis $\left(\mathrm{H}_{2}\right)$, and the fact that

$$
\lim _{m \rightarrow \infty} \operatorname{meas}\{|u|>m\}=0
$$

to arrive at

$$
\lim _{m \rightarrow \infty} \int_{\{m \leq|u| \leq m+k\}}|\nabla u|^{p(x)} S_{m}(u) d x=0 .
$$

TheOREM 3.3. Let hypotheses $\left(\mathrm{H}_{1}\right)-\left(\mathrm{H}_{2}\right)$ be satisfied. Then the nonlinear elliptic problem (1.1) has a unique renormalized solution.

Proof. It is known that under hypotheses $\left(\mathrm{H}_{1}\right)-\left(\mathrm{H}_{2}\right)$, there exists a renormalized solution of (1.1) (see for example [7]).

Uniqueness. Let $u$ and $v$ be two renormalized solutions of $(1.1)$ for the solution $u$. We take $S=S_{m}$ and $\varphi=S_{m}(v) T_{k}\left(T_{2 m}(u)-T_{2 m}(v)\right)$, where $m, k>0$. We get 


$$
\begin{aligned}
\int_{\Omega}|u|^{p(x)-2} u S_{m}(u) & S_{m}(v) T_{k}\left(T_{2 m}(u)-T_{2 m}(v)\right) d x \\
& +\int_{\partial \Omega} \gamma(u) S_{m}(u) S_{m}(v) T_{k}\left(T_{2 m}(u)-T_{2 m}(v)\right) d \sigma \\
& +\int_{\Omega}|\nabla u|^{p(x)-2} \nabla u \nabla\left(S_{m}(u) S_{m}(v) T_{k}\left(T_{2 m}(u)-T_{2 m}(v)\right)\right) d x \\
= & \int_{\Omega} f S_{m}(u) S_{m}(v) T_{k}\left(T_{2 m}(u)-T_{2 m}(v)\right) d x \\
& +\int_{\partial \Omega} g S_{m}(u) S_{m}(v) T_{k}\left(T_{2 m}(u)-T_{2 m}(v)\right) d \sigma .
\end{aligned}
$$

For the solution $v$, we take $S=S_{m}$ and $\varphi=S_{m}(u) T_{k}\left(T_{2 m}(u)-T_{2 m}(v)\right)$ to get

$$
\begin{aligned}
\int_{\Omega}|v|^{p(x)-2} v S_{m}(u) & S_{m}(v) T_{k}\left(T_{2 m}(u)-T_{2 m}(v)\right) d x \\
& +\int_{\partial \Omega} \gamma(v) S_{m}(u) S_{m}(v) T_{k}\left(T_{2 m}(u)-T_{2 m}(v)\right) d \sigma \\
& +\int_{\Omega}|\nabla v|^{p(x)-2} \nabla v \nabla\left(S_{m}(u) S_{m}(v) T_{k}\left(T_{2 m}(u)-T_{2 m}(v)\right)\right) d x \\
= & \int_{\Omega} f S_{m}(u) S_{m}(v) T_{k}\left(T_{2 m}(u)-T_{2 m}(v)\right) d x \\
& +\int_{\partial \Omega} g S_{m}(u) S_{m}(v) T_{k}\left(T_{2 m}(u)-T_{2 m}(v)\right) d \sigma .
\end{aligned}
$$

Subtracting the two equalities, we obtain

$$
\begin{aligned}
& \int_{\Omega}\left(|u|^{p(x)-2} u-|v|^{p(x)-2} v\right) S_{m}(u) S_{m}(v) T_{k}(u-v) d x+I(m, k) \\
& +\int_{\Omega}(\gamma(u)-\gamma(v)) S_{m}(u) S_{m}(v) T_{k}(u-v) d \sigma=0
\end{aligned}
$$

where

$$
I(m, k)=\int_{\Omega}\left(|\nabla u|^{p(x)-2} \nabla u-|\nabla v|^{p(x)-2} \nabla v\right) \nabla\left(S_{m}(u) S_{m}(v) T_{k}(u-v)\right) d x .
$$

By $\left(\mathrm{H}_{2}\right)$ we have

$$
\int_{\Omega}(\gamma(u)-\gamma(v)) S_{m}(u) S_{m}(v) T_{k}(u-v) d \sigma \geq 0
$$

Now, we prove that $I(m, k) \geq 0$. Set

$$
\begin{aligned}
\Omega_{m}^{1}=\{|u| \leq m,|v| \leq m\}, & \Omega_{m}^{2}=\{m<|u| \leq 2 m,|v| \leq m\}, \\
\Omega_{m}^{3}=\{m<|v| \leq 2 m,|u| \leq m\}, & \Omega_{m}^{4}=\{m<|u| \leq 2 m, m<|v| \leq 2 m\} .
\end{aligned}
$$


Let

$$
I(m, k)=\sum_{i=1}^{4} I_{i}(m, k)
$$

where

$$
\begin{aligned}
& I_{1}(m, k)=\int_{\Omega_{m}^{1}}\left(|\nabla u|^{p(x)-2} \nabla u-|\nabla v|^{p(x)-2} \nabla v\right) \nabla T_{k}(u-v) d x, \\
& I_{2}(m, k)=\int_{\Omega_{m}^{2}}\left(|\nabla u|^{p(x)-2} \nabla u-|\nabla v|^{p(x)-2} \nabla v\right) \nabla\left(\left(2-\frac{1}{m}|u|\right) T_{k}(u-v)\right) d x, \\
& I_{3}(m, k)=\int_{\Omega_{m}^{3}}\left(|\nabla u|^{p(x)-2} \nabla u-|\nabla v|^{p(x)-2} \nabla v\right) \nabla\left(\left(2-\frac{1}{m}|v|\right) T_{k}(u-v)\right) d x, \\
& I_{4}(m, k) \\
& =\int_{\Omega_{m}^{4}}\left(|\nabla u|^{p(x)-2} \nabla u-|\nabla v|^{p(x)-2} \nabla v\right) \nabla\left(\left(2-\frac{1}{m}|u|\right)\left(2-\frac{1}{m}|v|\right) T_{k}(u-v)\right) d x .
\end{aligned}
$$

First, the monotonicity of $\xi \mapsto|\xi|^{p(x)-2} \xi$ implies that

$$
I_{1}(m, k) \geq 0 \text {. }
$$

Secondly,

$$
\begin{aligned}
& I_{2}(m, k)=\int_{\Omega_{m}^{2}} \frac{-\operatorname{sign}(u)}{m}|\nabla u|^{p(x)} T_{k}(u-v) \\
& \quad+\int_{\Omega_{m}^{2}} \frac{\operatorname{sign}(u)}{m}|\nabla v|^{p(x)-2} \nabla v \nabla u T_{k}(u-v) d x \\
& \quad+\int_{\Omega_{m}^{2} \cap\{|u-v| \leq k\}}\left(|\nabla u|^{p(x)-2} \nabla u-|\nabla v|^{p(x)-2} \nabla v\right)\left(2-\frac{1}{m}|u|\right) \nabla(u-v) d x .
\end{aligned}
$$

On the one hand, by the properties of the truncated function $T_{k}$, we have

$$
\left.\left.\left|\int_{\Omega_{m}^{2}} \frac{-\operatorname{sign}(u)}{m}\right| \nabla u\right|^{p(x)} T_{k}(u-v)\left|\leq \frac{k}{m} \int_{\{m<|u| \leq 2 m\}}\right| \nabla u\right|^{p(x)} d x ;
$$

but

$$
\lim _{m \rightarrow \infty} \frac{1}{m} \int_{\{m<|u| \leq 2 m\}}|\nabla u|^{p(x)} d x=0,
$$

so

$$
\lim _{m \rightarrow \infty} \int_{\Omega_{m}^{2}} \frac{-\operatorname{sign}(u)}{m}|\nabla u|^{p(x)} T_{k}(u-v) d x=0 .
$$


On the other hand,

$$
\left.\left.\left|\int_{\Omega_{m}^{2}} \frac{\operatorname{sign}(u)}{m}\right| \nabla v\right|^{p(x)-2} \nabla v \nabla u T_{k}(u-v)\left|d x \leq \frac{k}{m} \int_{\Omega_{m}^{2}}\right| \nabla v\right|^{p(x)-1}|\nabla u| d x,
$$

i.e.

$$
\begin{aligned}
\left.\left|\int_{\Omega_{m}^{2}} \frac{\operatorname{sign}(u)}{m}\right| \nabla v\right|^{p(x)-2} \nabla v \nabla u T_{k}(u-v) \mid d x \\
\leq k \int_{\Omega_{m}^{2}}\left(\frac{1}{m}\right)^{\frac{1}{p^{\prime}(x)}}|\nabla v|^{p(x)-1}\left(\frac{1}{m}\right)^{\frac{1}{p(x)}}|\nabla u| d x,
\end{aligned}
$$

where $1 / p(\cdot)+1 / p^{\prime}(\cdot)=1$.

We use the Hölder type inequality (Proposition 2.1) to get

$$
\begin{aligned}
& \int_{\Omega_{m}^{2}}\left(\frac{1}{m}\right)^{\frac{1}{p^{\prime}(x)}}|\nabla v|^{p(x)-1}\left(\frac{1}{m}\right)^{\frac{1}{p(x)}}|\nabla u| d x \\
& \quad \leq\left(\frac{1}{p_{-}}+\frac{1}{p_{-}^{\prime}}\right)\left\|\left(\frac{1}{m}\right)^{\frac{1}{p^{\prime}(x)}}(\nabla v)^{p(x)-1}\right\|_{L^{p^{\prime}(\cdot)}\left(\Omega_{m}^{2,1}\right)}\left\|\left(\frac{1}{m}\right)^{\frac{1}{p(x)}} \nabla u\right\|_{L^{p(\cdot)}\left(\Omega_{m}^{2,2}\right)^{\prime}},
\end{aligned}
$$

where $\Omega_{m}^{2,1}=\{|v| \leq m\}$ and $\Omega_{m}^{2,2}=\{m<|u| \leq 2 m\}$.

Now, we use Proposition 2.2 to deduce that

$$
\begin{aligned}
&\left\|\left(\frac{1}{m}\right)^{\frac{1}{p^{\prime}(x)}}(\nabla v)^{p(x)-1}\right\|_{L^{p^{\prime}(\cdot)}\left(\Omega_{m}^{2,1}\right)} \\
& \leq \max \left\{\left(\rho_{L^{p^{\prime}(\cdot)}\left(\Omega_{m}^{2,1}\right)}\left(\left(\frac{1}{m}\right)^{\frac{1}{p^{\prime}(x)}}(\nabla v)^{p(x)-1}\right)\right)^{\frac{1}{p_{-}^{\prime}}},\right. \\
&\left.\left(\rho_{L^{p^{\prime}(\cdot)}\left(\Omega_{m}^{2,1}\right)}\left(\left(\frac{1}{m}\right)^{\frac{1}{p^{\prime}(x)}}(\nabla v)^{p(x)-1}\right)\right)^{\frac{1}{p_{+}^{\prime}}}\right\},
\end{aligned}
$$

and since

$$
\begin{aligned}
& \rho_{L^{p^{\prime}(\cdot)}\left(\Omega_{m}^{2,1}\right)}\left(\left(\frac{1}{m}\right)^{\frac{1}{p^{\prime}(x)}}(\nabla v)^{p(x)-1}\right)=\int_{\Omega_{m}^{2,1}}\left(\left(\frac{1}{m}\right)^{\frac{1}{p^{\prime}(x)}}|\nabla v|^{p(x)-1}\right)^{p^{\prime}(x)} d x \\
& =\frac{1}{m} \int_{\Omega_{m}^{2,1}}|\nabla v|^{p(x)} d x,
\end{aligned}
$$

by (3.13) there exists a constant $c_{1}$ independent of $m$ such that

$$
\left\|\left(\frac{1}{m}\right)^{\frac{1}{p^{\prime}(x)}}(\nabla v)^{p(x)-1}\right\|_{L^{p(\cdot)}\left(\Omega_{m}^{2,1}\right)} \leq c_{1} .
$$


By Proposition 2.2,

$$
\begin{aligned}
\|\left(\frac{1}{m}\right)^{\frac{1}{p(x)}} \nabla u & \|_{L^{p(\cdot)}\left(\Omega_{m}^{2,2}\right)} \\
\leq & \max \left\{\left(\rho_{L^{p(\cdot)}\left(\Omega_{m}^{2,2}\right)}\left(\left(\frac{1}{m}\right)^{\frac{1}{p(x)}} \nabla u\right)\right)^{\frac{1}{p_{-}}},\right. \\
& \left.\left(\rho_{L^{p(\cdot)}\left(\Omega_{m}^{2,2}\right)}\left(\left(\frac{1}{m}\right)^{\frac{1}{p(x)}} \nabla u\right)\right)^{\frac{1}{p_{+}}}\right\},
\end{aligned}
$$

and

$$
\begin{aligned}
\rho_{L^{p(\cdot)}\left(\Omega_{m}^{2,2}\right)}\left(\left(\frac{1}{m}\right)^{\frac{1}{p(x)}} \nabla u\right) & =\int_{\Omega_{m}^{2,2}}\left(\left(\frac{1}{m}\right)^{\frac{1}{p(x)}}|\nabla u|\right)^{p(x)} d x \\
& =\frac{1}{m} \int_{\Omega_{m}^{2,2}}|\nabla u|^{p(x)} d x .
\end{aligned}
$$

By using 3.2 we deduce that

$$
\lim _{m \rightarrow \infty}\left\|\left(\frac{1}{m}\right)^{\frac{1}{p(x)}} \nabla u\right\|_{L^{p(\cdot)}\left(\Omega_{m}^{2,2}\right)}=0 .
$$

Consequently, 3.18) and (3.19) imply that

$$
\lim _{m \rightarrow \infty} \int_{\Omega_{2}^{m}} \frac{\operatorname{sign}(u)}{m}|\nabla v|^{p-2} \nabla v \nabla u T_{k}(u-v)=0 .
$$

On the other hand,

$$
\left(2-\frac{1}{m}|u|\right) \chi_{\{m<|u| \leq 2 m\}} \geq 0,
$$

so

$$
\int_{\Omega_{2}^{m} \cap\{|u-v| \leq k\}}\left(|D u|^{p-2} \nabla u-|\nabla v|^{p-2} \nabla v\right)\left(2-\frac{1}{m}|u|\right) \nabla(u-v) \geq 0 .
$$

From (3.16), 3.20 and (3.21) we deduce that

$$
\lim _{m \rightarrow \infty} I_{2}(m, k) \geq 0 \text { for all } k>0 \text {. }
$$

Finally, we use the same method to find that

$$
\lim _{m \rightarrow \infty} I_{3}(m, k)+\lim _{m \rightarrow \infty} I_{4}(m, k) \geq 0 \quad \text { for all } k>0 .
$$

We now let $m \rightarrow \infty$ in 3.15 and divide the inequality by $k$ to conclude that

$$
\int_{\Omega}\left(|u|^{p(x)-2} u-|u|^{p(x)-2} v\right) \frac{T_{k}(u-v)}{k} d x \leq 0 .
$$


But

$$
\lim _{k \rightarrow 0} \frac{T_{k}(s)}{k}=\operatorname{sign}(s), \quad \operatorname{sing}(u-v)=\operatorname{sign}\left(|u|^{p(x)-2} u-|u|^{p(x)-2} v\right) ;
$$

therefore, by Fatou's lemma, we deduce that

$$
\int_{\Omega}\left|\left(|u|^{p(x)-2} u-|u|^{p(x)-2} v\right)\right| \leq 0 .
$$

This implies that $u=v$ a.e. in $\Omega$.

\section{References}

[1] A. Abassi, A. El Hachimi and A. Jamea, Entropy solutions to nonlinear Neumann problems with $L^{1}$-data, Int. J. Math. Statist. 2 (2008), no. S08, 4-17.

[2] R. A. Adams, Sobolev Spaces, Academic Press, 1975.

[3] F. Andreu, N. Igbida, J. Mazon and J. Toledo, Renormalized solutions for degenerate elliptic-parabolic problems with nonlinear dynamical boundary conditions, J. Differential Equations 244 (2008), 2764-2803.

[4] F. Andreu, J. M. Mazón, S. Segura de León and J. Toledo, Quasi-linear elliptic and parabolic equations in $L^{1}$ with nonlinear boundary conditions, Adv. Math. Sci. Appl. 7 (1997), 183-213.

[5] F. Andreu, N. Igbida, J. M. Mazón and J. Toledo, A degenerate elliptic-parabolic problem with nonlinear dynamical boundary conditions, Interfaces Free Bound. 78 (2006), 447-479.

[6] F. Andreu, N. Igbida, J. M. Mazón and J. Toledo, $L^{1}$ Existence and uniqueness results for quasi-linear elliptic equations with nonlinear boundary conditions, Ann. Inst. H. Poincaré Anal. Non Linéaire 24 (2007), 61-89.

[7] E. Azroul, A. Barbara, M. Benboubker, and S. Ouaro, Renormalized solutions for a $p(x)$-Laplacian equation with Neumann nonhomogeneous boundary conditions and $L^{1}$-data, Ann. Univ. Craiova Math. Computer Sci. Ser. 40 (2013), 9-22.

[8] M. Bendahmanea and P. Wittbold, Renormalized solutions for nonlinear elliptic equations with variable exponents and $L^{1}$ data, Nonlinear Anal. 70 (2009), 567-583.

[9] Ph. Bénilan, L. Boccardo, T. Gallouët, R. Gariepy, M. Pierre and J. L. Vázquez, An $L^{1}$ theory of existence and uniqueness of solutions of nonlinear elliptic equations, Ann. Scuola Norm. Sup. Pisa 22 (1995), 241-273.

[10] M. F. Betta, A. Mercaldo, F. Murat and M. M. Porzio, Uniqueness of renormalized solutions to nonlinear elliptic equations with a lower order term and right-hand side in $L^{1}(\Omega)$, ESAIM Control Optim. Calc. Var. 8 (2002), 239-272.

[11] D. Blanchard and F. Murat, Renormalized solutions of nonlinear parabolic problems with $L^{1}$ data: Existence and uniqueness, Proc. Roy. Soc. Edinburgh Sect. A 127 (1997), 1137-1152.

[12] H. Brezis, Analyse fonctionnelle, théorie et applications, Masson, Paris, 1983.

[13] Y. Chen, S. Levine, and M. Rao, Variable exponent, linear growth functionals in image restoration, SIAM J. Appl. Math. 66 (2006), 1383-1406.

[14] L. Diening, P. Hästö and A. Nekvinda, Open problems in variable exponent Lebesgue and Sobolev spaces, in: FSDONA04 Proceedings, P. Drabek and J. Rakosnik (eds.), Milovy, 2004, 38-58.

[15] L. Diening, P. Harjuleto, P. Hästö and M. Růžiča, Lebesgue and Sobolev Spaces with Variable Exponents, Lecture Notes in Math. 2017, Springer, 2011. 
[16] R. J. DiPerna and P.-L. Lions, On the Cauchy problem for Boltzmann equations: global existence and weak stability, Ann. of Math. 130 (1989), 321-366.

[17] A. El Hachimi and A. Jamea, Nonlinear parabolic problems with Neumann-type boundary conditions and $L^{1}$-data, Electron. J. Qualitat. Theory Differential Equations 2007, no. 27, 22 pp.

[18] X. Fan, On the sub-supersolution method for $p(x)$-Laplacian equations, J. Math. Anal. Appl. 330 (2007), 665-682.

[19] X. Fan and D. Zhao, On the spaces $L^{p(x)}(\Omega)$ and $W^{m, p(x)}(\Omega)$, J. Math. Anal. Appl. 263 (2001), 424-446.

[20] P. Harjulehto, P. Hästö and V. Latvala, Harnack's inequality for p(·)-harmonic functions with unbounded exponent $p$, J. Math. Anal. Appl. 352 (2009), 345-359.

[21] P. A. Hästö, The $p(x)$-Laplacian and applications, J. Anal. 15 (2007), 53-62.

[22] Y. Karagiorgos and N. Yannakakis, A Neumann problem involving the $p(x)$-Laplacian with $p=\infty$ in a subdomain, Adv. Calc. Var. 9 (2016), 65-76.

[23] J.-L. Lions, Quelques méthodes de résolution des problèmes aux limites non linéaires, Dunod, Paris, 1969.

[24] J. J. Manfredi, J. D. Rossi and J. M. Urbano, p(x)-Harmonic functions with unbounded exponent in a subdomain, Ann. Inst. H. Poincaré Anal. Non Linéaire 26 (2009), 2581-2595.

[25] F. Murat, Soluciones renormalizadas de EDP elipticas no lineales, preprint, Univ. Paris VI, 1993.

[26] F. Murat, Renormalized solutions of nonlinear elliptic equations with measure data, in: Journées équations aux dérivées partielles (Saint-Jean-de-Monts, 1998), exp. no. IX, Univ. Nantes, 1998, 4 pp.

[27] H. Redwane, Uniqueness of renormalized solutions for a class of parabolic equations with unbounded nonlinearities, Rend. Mat. Appl. 28 (2008), 189-200.

[28] M. Růžička, Electrorheological Fluids: Modeling and Mathematical Theory, Lecture Notes in Math. 1748, Springer, 2000.

[29] A. Siai, Nonlinear Neumann problems on bounded Lipschitz domains, Electron. J. Differential Equations 2005, no. 9, 16 pp.

[30] P. Wittbold and A. Zimmermann, Existence and uniqueness of renormalized solutions to nonlinear elliptic equations with variable exponent and $L^{1}$-data, Nonlinear Anal. 72 (2010), 2990-3008.

[31] C. Zhang, Entropy solutions for nonlinear elliptic equations with variable exponents, Electron. J. Differential Equations 2014, no. 92, 14 pp.

Ahmed Jamea, Jaouad Igbida

Département de Mathématiques

Centre Régional des Métiers

de l'Éducation et de Formation

El Jadida, Morocco

and

Laboratoire de Mathématiques Appliquées

à la Physique et Industrie

Faculté des Sciences

Université Chouaib Doukkali

El Jadida, Morocco

E-mail: a.jamea77@gmail.com

jigbida@yahoo.fr
Abderrahmane El Hachimi Département de Mathématiques

Faculté des Sciences Université Mohammed V Agdal, Rabat, Morocco E-mail: aelhachi@yahoo.fr 